\title{
Added Value of Arterial Spin-Labeling MR Imaging for the Differentiation of Cerebellar Hemangioblastoma from Metastasis
}

\author{
(D).M. Kang, (D).-H. Sohn, (D).-H. You, (D).G. Nam, (D) S.H. Choi, (D)T.J. Yun, (D) R.-E. Yoo, and (D).-h. Kim
}

\begin{abstract}
BACKGROUND AND PURPOSE: In adults with only cerebellar masses, hemangioblastoma and metastasis are the 2 most important differential diagnoses. Our aim was to investigate the added value of arterial spin-labeling MR imaging for differentiating hemangioblastoma from metastasis in patients with only cerebellar masses.
\end{abstract}

MATERIALS AND METHODS: This retrospective study included a homogeneous cohort comprising patients with only cerebellar masses, including 16 hemangioblastomas and 14 metastases. All patients underwent enhanced MR imaging, including arterial spin-labeling. First, the presence or absence of a hyperperfused mass was determined. Next, in the hyperperfused mass, relative tumor blood flow (mean blood flow in the tumor divided by blood flow measured in normal-appearing cerebellar tissue) and the size ratio (size in the arterial spin-labeling images divided by size in the postcontrast TIWI) were measured. To validate the arterial spin-labeling findings, 2 observers independently evaluated the conventional MR images and the combined set of arterial spin-labeling images.

RESULTS: All patients with hemangioblastomas and half of the patients with metastases presented with a hyperperfused mass $(P<.001)$. The size ratio and relative tumor blood flow were significantly larger for hemangioblastomas than for metastases $(P<.001$ and $P=.039$, respectively). The size ratio revealed excellent diagnostic power (area under the curve $=0.991$ ), and the relative tumor blood flow demonstrated moderate diagnostic power (area under the curve $=0.777)$. The diagnostic accuracy of both observers was significantly improved after the addition of arterial spin-labeling; the area under the curve improved from 0.574 to 0.969 ( $P<.001)$ for observer 2 and from 0.683 to $1(P<.001)$ for observer 2 .

CONCLUSIONS: Arterial spin-labeling imaging can aid in distinguishing hemangioblastoma from metastasis in patients with only cerebellar masses.

ABBREVIATIONS: $\mathrm{ASL}=$ arterial spin-labeling; $\mathrm{AUC}=$ area under the curve; $\mathrm{ROC}=$ receiver operating characteristic; $\mathrm{rTBF}=$ relative tumor blood flow

$\mathrm{H}$ emangioblastoma and metastasis are the 2 most important differential diagnoses of cerebellar masses in adults. Hemangioblastomas are benign tumors of vascular origin and are the second most common infratentorial parenchymal tumor, ac-

Received April 20, 2017; accepted after revision June 30.

From the Department of Radiology (K.M.K., C.-H.S., J.G.N., S.H.C., T.J.Y., R.-E.Y., J.-h.K.), Seoul National University Hospital, Seoul, Korea; Department of Radiology (C.-H.S., S.H.C.), Seoul National University College of Medicine, Seoul, Korea; and Department of Radiology (S.-H.Y.), Korea University Hospital, Seoul, Korea.

This study was supported by a grant from the Ministry of Science, ICT and Future Planning (grant No. NRF-2014M3C7A1046042)

Paper previously presented at: Annual Meeting of the American Society of Neuroadiology, April 22-27, 2017; Long Beach, California.

Please address correspondences to Chul-Ho Sohn, MD, PhD, Department of Radiology, Seoul National University Hospital, 101, Daehangno, Jongno-gu, Seoul 110-744, Korea; e-mail: neurorad63@gmail.com

-- Indicates open access to non-subscribers at www.ajnr.org

三 Indicates article with supplemental on-line table.

http://dx.doi.org/10.3174/ajnr.A5363 counting for $7 \%$ of posterior fossa tumors in adults. ${ }^{1}$ Metastases are the most common type of brain tumor, and posterior fossa metastases represent approximately $8.7 \%-10.9 \%$ of all brain metastases. ${ }^{2,3}$ Discriminating between hemangioblastoma and brain metastasis is important because their therapeutic approaches and prognoses are quite different. The standard treatment for hemangioblastoma is complete surgical resection. However, patients with a brain metastasis usually undergo surgery, stereotactic surgery, whole-brain radiation therapy, chemotherapy, or a combination of these. Furthermore, hemangioblastomas are associated with longer patient survival times, ${ }^{4}$ whereas brain metastases are associated with a poor prognosis. ${ }^{5}$ Because the frequency of metastasis increases with time, patient age is often helpful in distinguishing between these tumors but is not always reliable. In addition, although one-third of patients with cerebellar hemangioblastomas also have von Hippel-Lindau disease, ${ }^{6}$ a clinical history of von Hip- 
pel-Lindau disease may not be available at the time of initial presentation.

Hemangioblastoma is characterized by markedly increased vascularity ${ }^{7}$; therefore, angiographically dense tumor staining may suggest hemangioblastoma rather than metastasis. ${ }^{8}$ However, cerebral angiography is invasive and involves risks of complications, such as stroke. MR perfusion imaging can provide useful information about vascularization in hemangioblastoma. Previous studies using dynamic susceptibility contrast and dynamic contrast-enhanced MR perfusion imaging have reported increased vascular perfusion in hemangioblastomas. ${ }^{9,10}$

Arterial spin-labeling (ASL), unlike DSC and dynamic contrast-enhanced perfusion imaging, is a noninvasive MR perfusion technique that uses electromagnetic endogenous arterial water as a freely diffusible tracer instead of an exogenous MR imaging contrast agent. The utility of ASL perfusion imaging in the evaluation of the vascularity of brain tumors has been explored in several recent studies. ${ }^{11-14}$ One prior study differentiated hemangioblastomas from metastases on the basis of quantitative blood flow measurements using ASL. ${ }^{15}$ The authors reported that tumor blood flow was significantly higher in hemangioblastomas than in metastases. However, their study included a limited number of patients and examined metastatic tumors located primarily in the supratentorial region. Because metastasis usually presents with multiple enhancing supratentorial and infratentorial masses and $90 \%-95 \%$ of hemangioblastomas are in the posterior fossa, it is difficult to differentiate hemangioblastoma from metastasis in patients with only cerebellar masses in daily clinical practice.

Therefore, the aim of this study was to determine whether the addition of ASL imaging is useful for differentiating hemangioblastoma from metastasis in a homogeneous cohort of patients with only cerebellar masses and to validate the findings by investigating observer performance.

\section{MATERIALS AND METHODS}

This study was approved by our institutional review board, and informed consent was waived due to its retrospective nature.

\section{Subjects}

After a review of our radiology data base from October 2011 to October 2016, we identified 112 consecutive patients with cerebellar hemangioblastomas who satisfied the following inclusion criteria: 1) patients whose histopathologic diagnoses were confirmed as cerebellar hemangioblastomas or patients with von Hippel-Lindau disease having probable cerebellar hemangioblastomas, and 2) patients who did not have supratentorial mass lesions. For the patients with von Hippel-Lindau disease having probable cerebellar hemangioblastomas, the diagnosis of hemangioblastoma was made on the basis of radiologic evidence showing no other tumors with metastatic potential, including renal cell carcinomas, pheochromocytomas, or pancreatic neuroendocrine tumors. Of these patients, 96 were excluded for the following reasons: 1) lack of ASL images $(n=95)$, or 2$)$ no contrast-enhanced MR imaging $(n=1)$. The remaining 16 patients were included in this study. Ten of the 16 patients had von HippelLindau disease. Among the final 16 patients, 14 had pathologic confirmations of hemangioblastomas, and 2 had von Hippel-
Lindau disease; abdominal CT revealed 2 hemangiomas in the liver in 1 patient and multiple serous cystadenomas in the pancreas in the other.

We found 129 patients suspected of having cerebellar metastases who met the following inclusion criteria: 1) patients whose histopathologic diagnoses were confirmed as cerebellar metastases or patients whose diagnoses were made on the basis of their clinical history and follow-up imaging studies, ${ }^{16}$ and 2) patients who did not have supratentorial mass lesions. Of these patients, 115 were excluded on the basis of the following criteria: 1$)$ the presence of leptomeningeal seeding $(n=28), 2)$ history of previous radiation therapy or gamma knife surgery $(n=18), 3)$ coexisting acute infarction $(n=28), 4)$ no contrast-enhanced MR imaging $(n=1)$, and 5$)$ lack of ASL images $(n=40)$. Finally, 14 patients with cerebellar metastases confirmed by imaging follow-up $(n=12)$ or pathologic examination $(n=2)$ were included in the analysis.

\section{MR Imaging Protocol}

All patients underwent MR imaging with a 1.5T (Signa HDxt; GE Healthcare, Milwaukee, Wisconsin) or 3T (Discovery 750w; GE Healthcare) MR imaging scanner with an 8- or 32-channel head coil. Imaging sequences included FSE T2WI, contrast-enhanced spin-echo T1WI, and ASL images. MR imaging parameters were as follows: $\mathrm{TR}=467-567 \mathrm{~ms}, \mathrm{TE}=8-9 \mathrm{~ms}$, flip angle $=90^{\circ}$, and matrix $=320 \times 192$ for spin-echo T1WI; TR $=4850-5330 \mathrm{~ms}$, $\mathrm{TE}=92-127 \mathrm{~ms}$, flip angle $=90^{\circ}-142^{\circ}$, and matrix $=448 \times 256$ for FSE T2WI; and section thickness $=5 \mathrm{~mm}$ with a $1-\mathrm{mm}$ gap, FOV $=240 \times 240 \mathrm{~mm}$ for T1WI and T2WI. Contrast-enhanced T1WI was acquired after intravenous administration of gadobutrol (Gadovist; Bayer Schering Pharma, Berlin, Germany) at a dose of $0.1 \mathrm{mmol}$ per kilogram of body weight.

ASL images were acquired before administration of the contrast agent. ASL perfusion imaging was performed with a pseudocontinuous ASL pulse sequence, which significantly increases the flow-labeling efficacy in a single-coil setting. ${ }^{17,18}$ ASL images were acquired for 1.5 seconds of labeling followed by 1.5 seconds of labeling delay. Saturation pulses were used to suppress the background. ${ }^{19}$ The image acquisition consisted of a stack of interleaved 3D fast spin-echo spiral readouts, each of which lasted 4 $\mathrm{ms}$. Each spiral arm included 512 sampling points in $k$-space, and a total of 8 interleaves (arms) were acquired separately. The ASL parameters were as follows: $\mathrm{TR}=4446-4564 \mathrm{~ms}, \mathrm{TE}=9.4-9.9$ $\mathrm{ms}$, echo-train length $=1$, FOV $=240 \times 240 \mathrm{~mm}$, matrix $=$ $128 \times 128$, number of excitations $=3$, number of interleaved sections $=32$, section thickness $=5-6 \mathrm{~mm}$, intersection gap $=0$ $\mathrm{mm}$, and number of sections $=32-44$. The signal intensity change between the labeled image and the control image was fitted to a model from which a quantitative perfusion map of cerebral blood flow was obtained. ${ }^{20}$

\section{Image Analysis}

Two radiologists (a qualified neuroradiologist with 8 years of clinical experience and a third-year resident radiologist) who were blinded to the clinical and pathologic information independently reviewed the MR image sets in a random order. First, they reviewed the ASL images and determined the presence or absence of

AJNR Am J Neuroradiol 38:2052-58 Nov 2017 www.ajnr.org 2053 


\begin{tabular}{llllll}
\hline & \multicolumn{2}{c}{ Hemangioblastomas } & & \multicolumn{2}{c}{ Metastases } \\
\cline { 2 - 3 } \cline { 5 - 6 } & Intraobserver & Interobserver & & Intraobserver & Interobserver \\
\hline Size of an enhancing mass on postcontrast TIWI (a) & $0.900(0.739-0.964)$ & $0.975(0.931-0.991)$ & & $0.987(0.927-0.998)$ & $0.983(0.916-0.997)$ \\
Size of a hyperintense mass on ASL images (b) & $0.786(0.489-0.920)$ & $0.958(0.886-0.985)$ & & $0.973(0.850-0.995)$ & $0.935(0.705-0.998)$ \\
Size ratio (b/a) & $0.862(0.649-0.949)$ & $0.934(0.825-0.976)$ & & $0.730(0.049-0.948)$ & $0.801(-0.159-0.966)$ \\
rTBF & $0.824(0.567-0.935)$ & $0.643(0.246-0.858)$ & & $0.996(0.979-0.999)$ & $0.969(0.853-0.995)$ \\
\hline
\end{tabular}

${ }^{a}$ Data are intraclass correlation coefficient values, and the numbers in parentheses are $95 \% \mathrm{Cls}$.

a hyperperfused cerebellar mass in each patient. The hyperperfused mass was defined as a space-occupying hyperintense lesion compared with the appearance of normal gray matter on the CBF map from ASL imaging. In case of a hyperperfused mass, quantitative analyses were performed. The size of the mass was measured as the largest axial diameter on both the postcontrast T1WI and the CBF map from ASL imaging. The size ratio was calculated as the size on the CBF map divided by the size on the postcontrast T1WI.

Absolute tumor blood flow was calculated as the mean blood flow in an ROI, which was drawn over the hyperperfused mass on the section showing the largest cross-sectional area. ${ }^{15}$ Then, relative tumor blood flow (rTBF) was calculated by normalizing the absolute tumor blood flow to the blood flow measured in cortical gray matter with a normal appearance in the contralateral hemisphere based on methods in previous reports. ${ }^{15,21}$ An ROI of $>100 \mathrm{~mm}^{2}$ was selected in normal-appearing cortical gray matter. The reference ROI was placed in the same section as the one with the ROI for the tumor or the nearest section. In the case of tumors located across the midline, the reference ROI was placed in the cortical gray matter of the side in which the tumor was least present.

To assess the intraobserver reproducibility of the measurements, the qualified neuroradiologist with 8 years of clinical experience performed the size measurements and ROI placement twice at 2 -week intervals. The mean value of the repeat measurements was used to determine the tumor size, size ratio, and rTBF. Mean ROI sizes were $83.3 \pm 100 \mathrm{~mm}^{2}$ for hemangioblastomas and $204.4 \pm 75.6 \mathrm{~mm}^{2}$ for their corresponding contralateral gray matter; and $68.8 \pm 46.3 \mathrm{~mm}^{2}$ for metastases and $267.2 \pm 66.8$ $\mathrm{mm}^{2}$ for their corresponding contralateral gray matter.

\section{Observer Performance}

To investigate the added value of ASL imaging with conventional MR imaging for predicting hemangioblastomas, 2 qualified neuroradiologists (with 27 and 7 years of clinical experience, individually) who were blinded to the patients' histories and pathologic data independently reviewed 2 MR image sets-that is, the conventional image set including precontrast T1WI, T2WI, and postcontrast T1WI, and the combined ASL image set of the conventional image set and the CBF map derived from ASL perfusion imaging. For the conventional image set, hemangioblastoma diagnoses were made according to the following findings: a markedly enhancing mural nodule with a large surrounding cyst, a solid enhancing nodule, or the presence of large draining and feeding vessels within the periphery and a solid nodule. ${ }^{8,22}$ For the combined ASL image set, the observers were provided with the predictive findings, the measurement results (median and ranges) of each tumor, and the
Table 2: Results of the comparative analyses ${ }^{a}$

\begin{tabular}{lccr} 
& $\begin{array}{c}\text { Hemangioblastomas } \\
(\boldsymbol{n}=16)\end{array}$ & $\begin{array}{c}\text { Metastases } \\
(\boldsymbol{n}=\mathbf{7})\end{array}$ & $\boldsymbol{P}$ Value $^{\mathrm{b}}$ \\
\hline Size ratio $^{\mathrm{b}}$ & $1.4(0.95-3.64)$ & $0.80(0.60-0.98)$ & $<.001$ \\
rTBF $^{\mathrm{b}}$ & $8.18(1.95-17.15)$ & $2.44(1.88-14.36)$ & .039 \\
\hline
\end{tabular}

a Size, rTBF, and size ratio data are presented as median values, and the numbers in parentheses are the lowest and highest values.

${ }^{\mathrm{b}}$ Mann-Whitney $U$ test comparing the size ratio and rTBF between hemangioblastomas and metastases.

diagnostic performances of rTBF and the size ratio, which were acquired from the above image analyses.

For each set, the observers were asked to predict the presence of a hemangioblastoma according to the provided criteria, and they recorded their confidence levels with the following 5-point scale: 1 , definitely metastasis; 2 , probably metastasis; 3 , indeterminate lesion; 4, probably hemangioblastoma; and 5, definitely hemangioblastoma.

\section{Statistical Analysis}

Intra- and interobserver reproducibility for the size, size ratio, and rTBF were assessed by calculating intraclass correlation coefficients. ${ }^{23}$ The Fisher exact test was used to compare the frequencies of a hyperperfused mass between hemangioblastomas and metastases. The difference in sizes between the postcontrast T1WI and the CBF map was evaluated with the Wilcoxon signed rank test for hemangioblastomas and metastases. The size ratios and rTBF values were compared between hemangioblastomas and metastases with the Mann-Whitney test. A receiver operating characteristic (ROC) curve analysis was performed to evaluate the diagnostic performance of the size ratio and rTBF for differentiating hemangioblastoma from cerebellar metastasis. In addition, a pair-wise comparison of the ROC curves was performed to compare the diagnostic accuracies of the size ratio and rTBF.

For observer performance, interobserver variability regarding the confidence level for diagnosing hemangioblastoma was assessed with $\kappa$ statistics. The diagnostic performance of each observer was assessed with an ROC curve analysis. To compare the diagnostic accuracy before and after evaluating the ASL images, we performed a pair-wise comparison of the ROC curves. We used $95 \%$ confidence intervals to express the statistical precision of the results. Sensitivity, specificity, and diagnostic accuracy were calculated for both observers on the assumption that a confidence level of 4 or higher was positive for the diagnosis of hemangioblastoma. Under this assumption, the McNemar test was used to compare the overall accuracy before and after evaluating additional ASL images.

Statistical analyses were performed with MedCalc for Windows (Version 9.3.0.0; MedCalc Software, Mariakerke, Belgium). $P<.05$ was considered statistically significant. 

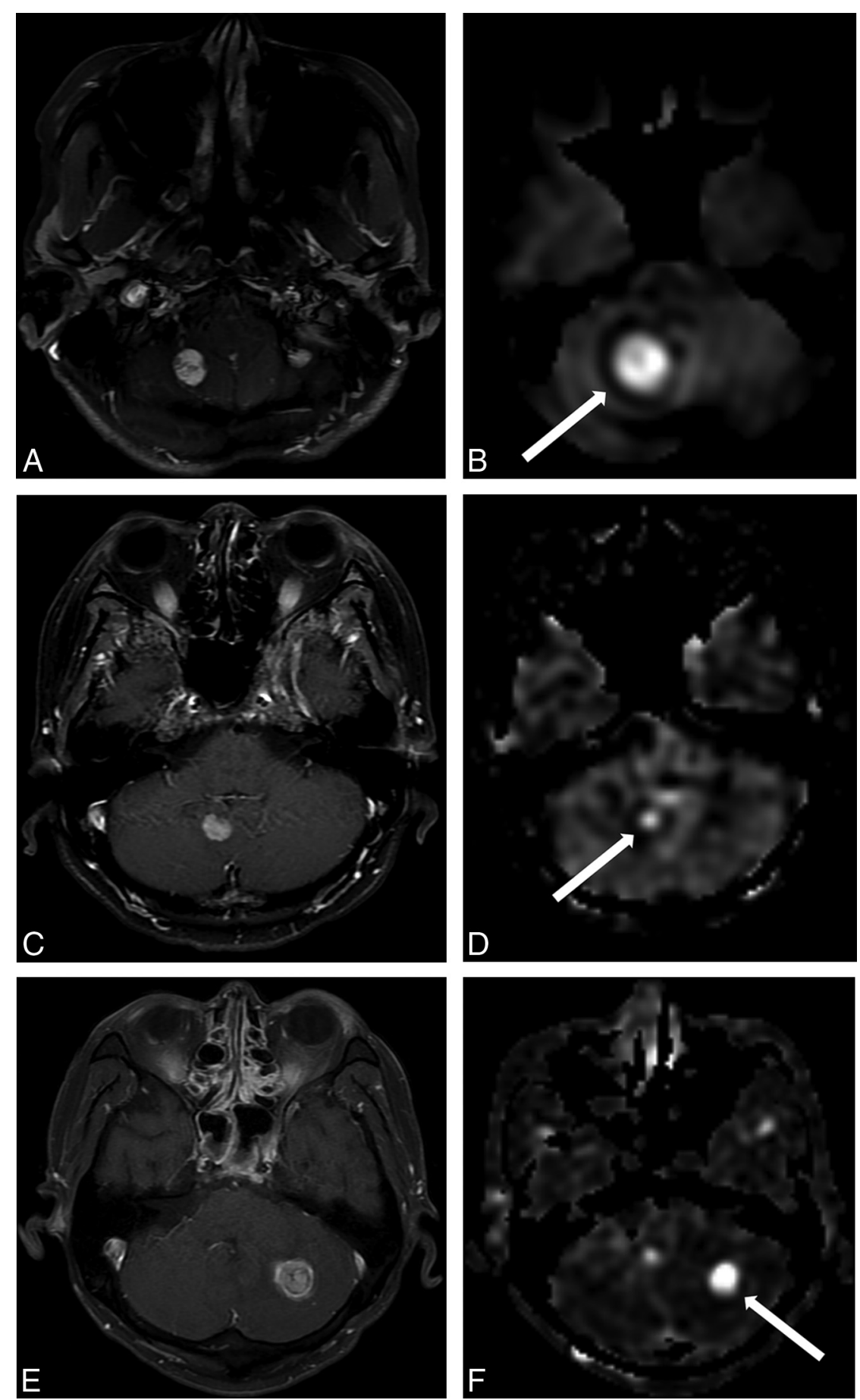

FIG 1. Contrast-enhanced TIWI ( $A$ and $C$ ) and CBF maps derived from ASL imaging ( $B$ and $D)$ for 2 representative cases. $A$ and $B, A$ cerebellar hemangioblastoma in a 36-year-old woman with a high size ratio of 1.92 and $\mathrm{rTBF}$ of 6.16. $C$ and $D$, A cerebellar metastasis in a 42-year-old woman with breast cancer with a relatively low size ratio of 0.71 and $\mathrm{rTBF}$ of 2.1. $E$ and $F$, A cerebellar metastasis in a 56-year-old man with renal cell carcinoma with a size ratio of 0.82 and rTBF of 14.36 .

\section{RESULTS}

Patients with cerebellar hemangioblastomas included 11 men (mean age, 41.8 years; range, $20-71$ years) and 5 women (mean age, 44.2 years; range, $36-62$ years). Patients with cerebellar metastases included 8 men (mean age, 60.6 years; range, $41-76$ years) and 6 women (mean age, 47.3 years; range, $40-58$ years). The primary types of cancer in these patients were as follows: lung cancer $(n=6)$, breast cancer $(n=5)$, gastric cancer $(n=1)$, colon cancer $(n=1)$, and renal cell carcinoma $(n=1)$. Three of the 16 patients with hemangioblastomas and 2 of the 14 patients with metastases had $>2$ masses. In patients with multiple hyperperfused masses, the largest representative mass was included in the following quantitative analyses.

\section{Image Analysis for Differentiating Hemangioblastoma from \\ Metastasis}

The presence of at least 1 hyperperfused mass in the cerebellum was observed in all 16 patients with hemangioblastomas and in 7 of the $14(50 \%)$ patients with metastases by both reviewers, with $100 \%$ agreement. There was a significant difference in the frequency of hyperperfused cerebellar masses between patients with hemangioblastomas and those with metastases $(P=.0002)$. Hyperperfused cerebellar metastases were observed in patients with lung cancer $(n=2)$, breast cancer $(n=2)$, gastric cancer $(n=1)$, colon cancer $(n=1)$, and renal cell carcinoma $(n=1)$. The other lung $(n=4)$ and breast cancer $(n=3)$ metastases did not demonstrate hyperperfusion. The analyses described below were performed on the hyperperfused masses (16 hemangioblastomas and 7 metastases).

The intraclass correlation coefficient values, with $95 \%$ CIs, were calculated for the size, size ratio, and rTBF data, as shown in Table 1. For both hemangioblastomas and metastases, the intra- and interobserver agreements were fair to excellent for the size, size ratio, and rTBF values.

The Wilcoxon signed rank test of the paired samples revealed that the measured size of hemangioblastomas was significantly larger on the CBF map (median, $16.04 \mathrm{~mm}$; range, $6.75-46.27 \mathrm{~mm}$ ) than on the postcontrast T1WI (median, 20.12 $\mathrm{mm}$; range, 8.87-33.70 $\mathrm{mm} ; P<.001)$. Conversely, the measured size of hyperperfused metastases was significantly larger on the postcontrast T1WI (median, $25.02 \mathrm{~mm}$; range, $12.47-36.24 \mathrm{~mm}$ ) than on the CBF map (median, $20.12 \mathrm{~mm}$; range, 8.87-33.70 mm; $P=.016$ ). The Mann-Whitney test revealed that the size ratio of hemangioblastomas was significantly larger than that of metastases $(P<.001)$. Furthermore, the rTBF of hemangioblastomas was significantly higher than that of metastases $(P=.039)$. The results are summarized in Table 2. Representative images are shown in Fig 1.

AJNR Am J Neuroradiol 38:2052-58 Nov 2017 www.ajnr.org 


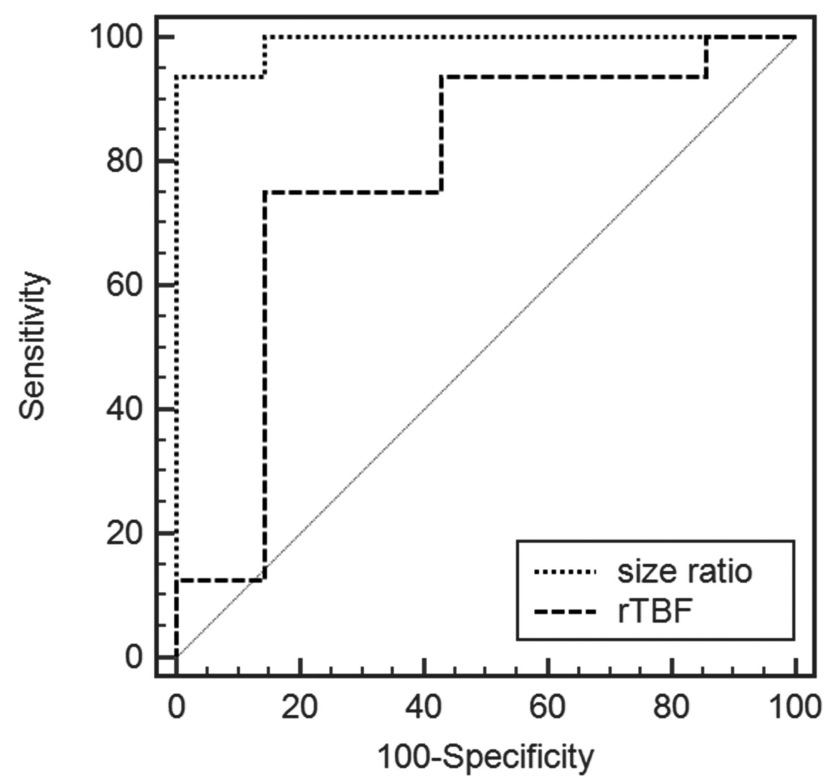

FIG 2. ROC curves used to differentiate hemangioblastomas from metastases by comparing size ratio and rTBF values. The AUC values for the rTBF and size ratio were 0.777 and 0.991 , respectively.

According to the ROC analysis, the area under the curve (AUC) was 0.991 (95\% CI, 0.83-1.000) for the size ratio and 0.777 (95\% CI, 0.557-0.922) for the rTBF. A size ratio of $>0.98$ could differentiate hemangioblastomas from hyperperfused metastases with a sensitivity of $93.7 \%(15 / 16)$, a specificity of $100 \%(7 / 7)$, and an accuracy of $91 \%(40 / 44)$. A rTBF of $>3.78$ corresponded to a sensitivity of $75 \%(12 / 16)$, a specificity of $85.7 \%(6 / 7)$, and an accuracy of 78.3\% (18/23) (Fig 2). Although the AUC value for the size ratio (0.991) was higher than for the rTBF (0.777), they were not significantly different in the comparison of ROC curves $(P=$ $.062)$.

There were no significant differences in the frequencies of hemangioblastomas and metastases between patients examined with $3 \mathrm{~T}$ and $1.5 \mathrm{~T}$ magnets $(P=.657)$. Nine of the 16 patients with hemangioblastomas and 8 of the 14 patients with metastases underwent MR imaging at 1.5T, and the others underwent MR imaging at 3T. Among 7 patients with hyperperfused metastases, 5 underwent MR imaging at 1.5T. Detailed results for the size ratio and $\mathrm{rTBF}$ data regarding magnetic strength are summarized in the On-line Table.

\section{Observer Performance}

Interobserver agreement was improved from poor $(\kappa=0.384$; 95\% CI, 0.217-0.551) to excellent ( $\kappa=0.911$; 95\% CI, 0.777-1) after the addition of ASL imaging to conventional image sets. The diagnostic accuracy in the differentiation of hemangioblastomas from metastases was significantly improved for both observers after they reviewed the ASL images: The AUC improved from 0.574 to $0.969(P<.001)$ for observer 1 and from 0.683 to $1(P<$ .001 ) for observer 2 (Table 3 and Fig 3 ). The McNemar test revealed that the overall accuracy significantly improved for both observers after the addition of the ASL images (observer 1, $P=$ .0005 ; observer $2, P=.022$ ). Additional ASL image interpretation enabled observers to correct several diagnostic errors (observer 1 ,
Table 3: Diagnostic predictive values for the differentiation of hemangioblastomas from metastases ${ }^{a}$

\begin{tabular}{lcc}
\hline Reading Session and Statistic & Observer 1 & Observer 2 \\
\hline Conventional image set & & \\
AUC & & \\
Sensitivity (\%) & $18.8(3 / 16)$ & $43.7(7 / 16)$ \\
Specificity (\%) & $100(14 / 14)$ & $92.9(13 / 14)$ \\
Accuracy (\%) & $57(17 / 30)$ & $67(20 / 30)$ \\
Combined ASL image set & & \\
AUC & & $1.000(0.884-1.000)$ \\
Sensitivity (\%) & $93.7(15 / 16)$ & $100(16 / 16)$ \\
Specificity (\%) & $100(14 / 14)$ & $100(14 / 14)$ \\
Accuracy (\%) & $97(29 / 30)$ & $100(30 / 30)$ \\
$P$ value for pair-wise & $<.001$ & $<.0001$ \\
$\quad$ comparison of ROC & & \\
curves & & .022 \\
$P$ value calculated with the & $<.001$ & \\
McNemar test for the & & \\
comparison of accuracy & & \\
\hline
\end{tabular}

a Unless otherwise indicated, the numbers in parentheses are raw data. Sensitivity, specificity, and accuracy were calculated under the assumption that a confidence score of 4 or higher was considered positive for the diagnosis of hemangioblastoma. ${ }^{\mathrm{b}}$ Data in parentheses are $95 \% \mathrm{Cls}$.

$n=12$; observer $2, n=13$ ) made on the basis of conventional image interpretation alone.

\section{DISCUSSION}

Our study investigated the added diagnostic value of ASL imaging for the differentiation of hemangioblastomas from metastases in a homogeneous cohort of patients with only cerebellar masses. All patients with hemangioblastomas had at least 1 hyperperfused mass, while only half of the patients with metastases presented with a hyperperfused mass. The size ratios and rTBF of the masses were measured for differential diagnoses between hemangioblastomas and metastases for the hyperperfused cerebellar masses. The values of both variables were significantly higher in hemangioblastomas than in metastases. The size ratio revealed excellent diagnostic power (AUC $=0.991$ ), and rTBF demonstrated moderate diagnostic power $(\mathrm{AUC}=0.777)$. In addition, we investigated the 2 observers' performances to validate the use of ASL for distinguishing hemangioblastomas from metastases in patients with only cerebellar masses. Both the diagnostic accuracy of the observers and the interobserver agreement were significantly improved after the addition of ASL images.

A prior study with ASL reported that rTBF was significantly higher for hemangioblastomas than for metastases. ${ }^{15}$ However, the study included only 5 hemangioblastomas, and 10 of 14 (71\%) metastases were in the supratentorial region. In addition, there was no information regarding visually assessed signal intensities - that is, the prevalence of a hyperperfused mass in the respective tumors. One of strengths of this study is that we included a homogeneous cohort of patients with only cerebellar masses for both hemangioblastomas and metastases. In addition, the frequency of the presence of hyperperfused masses in the 2 tumor types was assessed. In contrast to the patients with metastases, no patients with hemangioblastomas had hypo- or isointense cerebellar masses. Therefore, when a patient has an enhancing mass in the cerebellum that does not show hyperperfusion on ASL imaging, the diagnosis might not be hemangioblastoma.

The rTBF and size ratio of hyperperfused masses aided in differentiating hemangioblastomas and metastases. Our rTBF results are like those of a previous study. ${ }^{15}$ They reported that the 

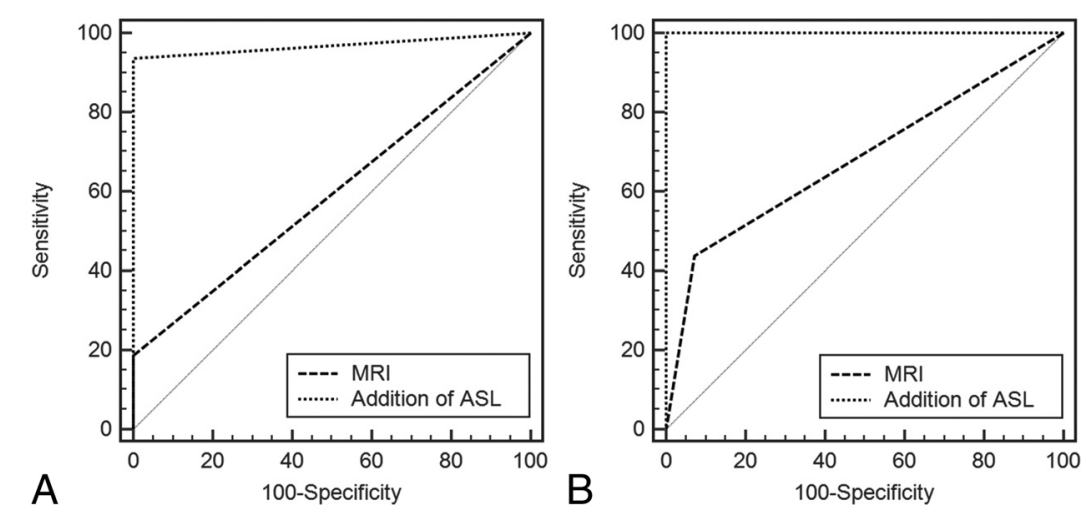

FIG 3. ROC curves used to differentiate hemangioblastomas from metastases before and after the addition of ASL MR images for both observers. A, For observer 1, the AUC was significantly improved after additional review of the ASL MR images (from 0.594 to 0.969). B, For observer 2, the AUC was also significantly improved after this review (from 0.683 to 1).

mean rTBF was $7.96 \pm 3.12$ in hemangioblastomas and $2.98 \pm$ 3.91 in metastases, and its diagnostic accuracy was $84.2 \%$, with a cutoff value of 3.3. In our study, the median rTBF was 8.18 (interquartile range, 4.10-12.19) in hemangioblastomas and 2.44 (interquartile range, 2.04-3.73) in metastases, and the diagnostic accuracy was $78.3 \%$, with a cutoff value of 3.78 .

However, we also investigated the diagnostic performance of size ratio, and it revealed a better accuracy of $91 \%$, with a cutoff value of 0.98 . In addition, the multivariable analysis revealed that size ratio was the only independent predictor of a differential diagnosis. Therefore, the size ratio seems to be useful for differentiating hemangioblastoma from metastasis. For example, a metastasis from renal cell carcinoma mimicked a hemangioblastoma due to its very high rTBF (16.2), like the findings for hemangioblastomas reported in the previous study. ${ }^{15}$ However, in our study, an accurate diagnosis of a metastasis from renal cell carcinoma was made when it was based on the low size ratio of 0.82 , which took precedence over a high rTBF of 14.36 (Fig 1).

The size ratio determined by ASL imaging suggests that hemangioblastomas appear to become enlarged or "bloom" in ASL sequences. Because a hemangioblastoma is composed of a cluster of thin-walled, tightly packed blood vessels lined with endothelial cells, it may be identified as a dense tumor nodule or as a heterogeneous network of tangled vessels fed by dilated artery draining veins in angiographs. ${ }^{8}$ Therefore, we presumed that the "blooming" appearance represented the emphasized bright signal intensities, which might be attributed to delayed filling and flow stagnation in the tumor.

Observer performance was evaluated to validate the findings based on ASL imaging. The diagnostic accuracy for differentiating hemangioblastomas from metastases was significantly improved after additional review of the CBF map from ASL imaging for both observers. Observer 1 made 1 error after viewing the additional ASL images. On review, the size ratio had been overlooked in this case. The addition of ASL images increased the $\kappa$ value between observer 1, who was an experienced radiologist with 28 years of clinical experience, and the other observer, who had 6 years of clinical experience. This finding suggests that not only diagnostic accuracy but also interobserver variation can be improved by the addition of ASL images.
When the clinical findings are noncontributory and hemangioblastomas appear as solid contrast-enhancing masses with peritumoral edema, conventional MR imaging cannot distinguish them from metastases. ${ }^{10}$ In such cases, markedly increased vascularity visible on cerebral angiographs may suggest hemangioblastoma rather than metastasis. ${ }^{8}$ However, a non-negligible neurologic complication rate of up to $1.3 \%$ has been reported. ${ }^{24-26}$ In addition, preoperative embolization is not generally used. ${ }^{27}$ Therefore, better diagnostic accuracy with MR imaging is crucial. Our results suggest that the addition of ASL imaging could provide practical information to differentiate hemangioblastoma from metastasis in patients with only enhancing cerebellar masses. When enhancing cerebellar masses do not show hyperperfusion on ASL imaging, the possibility of hemangioblastoma is minimal. When enhancing cerebellar masses reveal hyperperfusion, the size ratio can provide strong diagnostic power. If the masses appear larger on the contrast-enhanced T1WI than on the CBF map, then the possibility of hemangioblastoma is minimal. In patients with impaired renal function, rTBF alone may aid in differentiating hemangioblastoma from metastasis using noncontrast conventional MR imaging with additional ASL imaging.

There are several limitations of this study. First, this was a retrospective study, and the sample size was small. Therefore, the same population had to be used to validate the suggested ASL findings. In addition, because of the small sample size, the comparison of ROC curves did not reveal a significant difference between the AUC values for the size ratio and $\operatorname{rTBF}(P=.062)$. Despite this limitation, we included a relatively larger number of patients compared with several previous studies using MR perfusion imaging., ${ }^{9,10,15}$ Furthermore, we included a homogeneous cohort of patients with only cerebellar masses. Second, ASL imaging was performed with 2 different MR imaging scanners with different magnetic field strengths. However, we used the normalized rTBF values to minimize the influence of these differences. Although, statistical comparison could not be performed due to the small sample size, a subgroup analysis still revealed that the size ratio and rTBF values were larger for hemangioblastomas than for metastases.

\section{CONCLUSIONS}

The addition of ASL imaging to conventional MR imaging may be a reliable and noninvasive method to increase diagnostic accuracy and interobserver variation for the differentiation of hemangioblastoma from metastasis in patients with only cerebellar masses. Combining size ratio and rTBF data could aid in providing correct diagnoses.

\section{ACKNOWLEDGMENTS}

The authors appreciate the statistical advice from the Medical Research Collaborating Center at the Seoul National University Hospital.

AJNR Am J Neuroradiol 38:2052-58 Nov 2017 www.ajnr.org 


\section{REFERENCES}

1. Chaudhry AP, Montes M, Cohn GA. Ultrastructure of cerebellar hemangioblastoma. Cancer 1978;42:1834-50 Medline

2. Yoshida S, Takahashi H. Cerebellar metastases in patients with cancer. Surg Neurol 2009;71:184-87; discussion 187 CrossRef Medline

3. Kanner AA, Suh JH, Siomin VE, et al. Posterior fossa metastases: aggressive treatment improves survival. Stereotact Funct Neurosurg 2003;81:18-23 CrossRef Medline

4. Catapano D, Muscarella LA, Guarnieri V, et al. Hemangioblastomas of central nervous system: molecular genetic analysis and clinical management. Neurosurgery 2005;56:1215-21; discussion 1221 CrossRef Medline

5. Alexandru D, Bota D, Linskey M. Epidemiology of central nervous system metastases. In: Kim DG, Lunsford LD, eds. Current and Future Management of Brain Metastasis. Basel: Karger Publishers; 2012:13-29

6. Richard S, Campello C, Taillandier L, et al. Haemangioblastoma of the central nervous system in von Hippel-Lindau disease: French VHL Study Group. J Intern Med 1998;243:547-53 CrossRef Medline

7. Berkman RA, Merrill MJ, Reinhold WC, et al. Expression of the vascular permeability factor/vascular endothelial growth factor gene in central nervous system neoplasms. J Clin Invest 1993;91:153-59 CrossRef Medline

8. Ho VB, Smirniotopoulos JG, Murphy FM, et al. Radiologic-pathologic correlation: hemangioblastoma. AJNR Am J Neuroradiol 1992; 13:1343-52 Medline

9. Cha J, Kim ST, Nam D-H, et al. Differentiation of hemangioblastoma from metastatic brain tumor using dynamic contrast-enhanced MR imaging. Clin Neuroradiol 2016 Mar 7. [Epub ahead of print] CrossRef Medline

10. She D, Yang X, Xing Z, et al. Differentiating hemangioblastomas from brain metastases using diffusion-weighted imaging and dynamic susceptibility contrast-enhanced perfusion-weighted MR imaging. AJNR Am J Neuroradiol 2016;37:1844-50 CrossRef Medline

11. Yoo RE, Yun TJ, Cho YD, et al. Utility of arterial spin labeling perfusion magnetic resonance imaging in prediction of angiographic vascularity of meningiomas. J Neurosurg 2016;125:536-43 CrossRef Medline

12. Sunwoo L, Yun TJ, You S-H, et al. Differentiation of glioblastoma from brain metastasis: qualitative and quantitative analysis using arterial spin labeling MR imaging. PLoS One 2016;11:e0166662 CrossRef Medline

13. Warmuth C, Gunther M, Zimmer C. Quantification of blood flow in brain tumors: comparison of arterial spin labeling and dynamic susceptibility-weighted contrast-enhanced MR imaging. Radiology 2003;228:523-32 CrossRef Medline

14. Noguchi T, Yoshiura T, Hiwatashi A, et al. Perfusion imaging of brain tumors using arterial spin-labeling: correlation with histo- pathologic vascular density. AJNR Am J Neuroradiol 2008;29:688-93 CrossRef Medline

15. Yamashita K, Yoshiura T, Hiwatashi A, et al. Arterial spin labeling of hemangioblastoma: differentiation from metastatic brain tumors based on quantitative blood flow measurement. Neuroradiology 2012;54:809-13 CrossRef Medline

16. Jeon JY, Choi JW, Roh HG, et al. Effect of imaging time in the magnetic resonance detection of intracerebral metastases using single dose gadobutrol. Korean J Radiol 2014;15:145-50 CrossRef Medline

17. Dai W, Garcia D, de Bazelaire C, et al. Continuous flow-driven inversion for arterial spin labeling using pulsed radio frequency and gradient fields. Magn Reson Med 2008;60:1488-97 CrossRef Medline

18. Garcia D, De Bazelaire C, Alsop D. Pseudo-continuous flow driven adiabatic inversion for arterial spin labeling. In: Proceedings of the International Society for Magnetic Resonance in Medicine, Miami, Florida. May 7-13, 2005;13:37

19. Mani S, Pauly J, Conolly S, et al. Background suppression with multiple inversion recovery nulling: applications to projective angiography. Magn Reson Med 1997;37:898-905 CrossRef Medline

20. Yoo RE, Choi SH, Cho HR, et al. Tumor blood flow from arterial spin labeling perfusion MRI: a key parameter in distinguishing high-grade gliomas from primary cerebral lymphomas, and in predicting genetic biomarkers in high-grade gliomas. J Magn Reson Imaging 2013;38:852-60 CrossRef Medline

21. Lehmann P, Monet P, de Marco G, et al. A comparative study of perfusion measurement in brain tumours at 3 Tesla MR: arterial spin labeling versus dynamic susceptibility contrast-enhanced MRI. Eur Neurol 2010;64:21-26 CrossRef Medline

22. Leung RS, Biswas SV, Duncan M, et al. Imaging features of von Hippel-Lindau disease. Radiographics 2008;28:65-79 CrossRef Medline

23. Kang KM, Lee JM, Yoon JH, et al. Intravoxel incoherent motion diffusion-weighted MR imaging for characterization of focal pancreatic lesions. Radiology 2014;270:444-53 CrossRef Medline

24. Willinsky RA, Taylor SM, TerBrugge K, et al. Neurologic complications of cerebral angiography: prospective analysis of 2,899 procedures and review of the literature. Radiology 2003;227:522-28 CrossRef Medline

25. Dawkins A, Evans A, Wattam J, et al. Complications of cerebral angiography: a prospective analysis of 2,924 consecutive procedures. Neuroradiology 2007;49:753-59 CrossRef Medline

26. Thiex R, Norbash A, Frerichs K. The safety of dedicated-team catheter-based diagnostic cerebral angiography in the era of advanced noninvasive imaging. AJNR Am J Neuroradiol 2010;31:230-34 CrossRef Medline

27. Montano N, Doglietto F, Pedicelli A, et al. Embolization of hemangioblastomas. J Neurosurg 2008;108:1063-64; author reply 1064-65 CrossRef Medline 